\title{
The different equations of motion of the central line of a slender vortex filament and their use to study perturbed vortices
}

\author{
D. Margerit and J-P. Brancher
}

LEMTA (CNRS UMR 7563)

2 avenue de la forêt de Haye BP 160

54504 Vandoeuvre les Nancy, France.

dmargeri@ensem.u-nancy.fr

\begin{abstract}
A comparison between the equation of motion of the central line of a slender vortex filament deduced from a matched asymptotic expansion[1] and the expansion of the equation of motion of the ad-hoc cut-off methods[2] with the cut-off length as the small asymptotic parameter is performed. It justifies the cut-off methods and gives the link between the cut-off lengths and the thickness of a viscous or inviscid vortex with an axial velocity component. The asymptotic equation of motion for an open filament is then simplified in case of a perturbed straight filament and different regimes are displayed. They depend of relatives values of the amplitude of the perturbation and the small thickness of the filament.
\end{abstract}

\section{Introduction}

It is well known that the structure of flows often exhibits concentration of vorticity in the form of vortex sheets or of vortex filaments. They can be seen in aircraft wakes, jets, boundary layer, Direct Numerical Simulations of turbulent flows, tornados,...

There are a considerable number of publications about the motion of a vortex filament and its stability. All field of methods have been used : formal asymptotic and linear stability analyses, direct numerical simulations, experimental investigations,...Theoretical studies have been performed either with Navier Stokes equations or simplified ones. In the later case, the field that covers the result of the study or its validity depends of the field of validity of the simplified equation that is used. This domain of validity needs to be given.

Here, a general dimensionless description of a slender vortex filament is given as an introduction to all discussion. A number of ad-hoc equation of motion due to ad-hoc regularization of the singular line BiotSavart integral are given and compared with the results of a systematic matched asymptotic expansions. It is performed both for a closed vortex filament and an open one. Our first main result is to give a relation which makes a link between ad-hoc and "exact" asymptotic approaches. The asymptotic equation of motion for an open filament is then simplified in case of a perturbed straight filament and different regimes are displayed. They depend of respective values of the amplitude of the perturbation and the small thickness of the filament. This is our second main result, that is sum up on a diagram showing the domain of validity of the various simplified equations.

\section{Description of a vortex filament}

A slender vortex filament of thickness $\delta$ is a solenoidal field of vorticity $\boldsymbol{\omega}(\mathbf{x}, t)$ which is non-zero only in the neighbourhood of a three-dimensional curve $\mathcal{C}$, called the central line. This curve is described parametrically (Fig.1) with the use of a function $\mathbf{X}=\mathbf{X}(s, t)$, which denotes a point on the curve as a function of the parameter $s$ and time $t$. Either $s \in[-\pi, \pi[$ for a vortex ring that has a closed central curve, or $s \in[-\infty, \infty[$ 


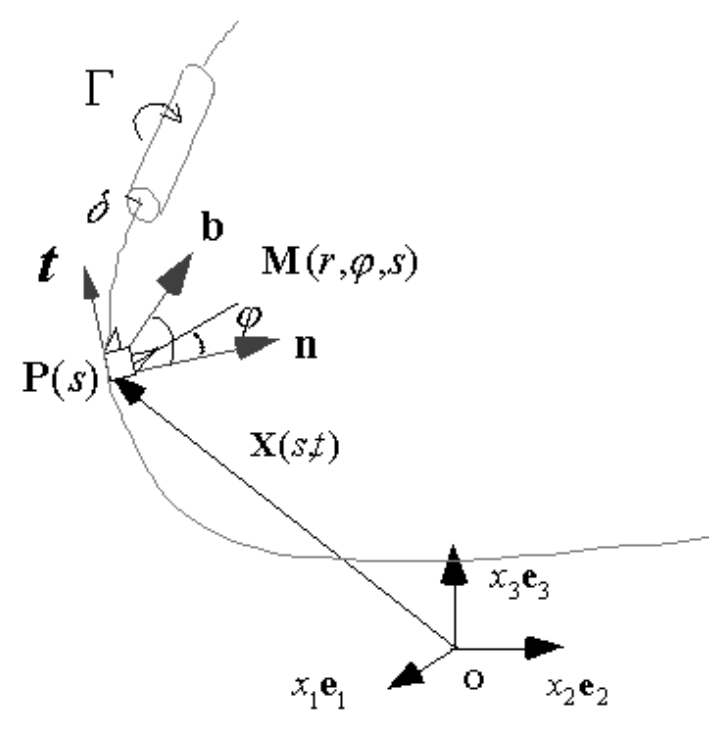

Figure 1: The central curve and the local co-ordinates of the vortex filament.

for an open central line. The local torsion of $\mathcal{C}$ is called $T$ and $K$ is the local curvature. The function $\sigma$ is defined by

$$
\sigma(s, t)=|\partial \mathbf{X} / \partial s|
$$

where || is the usual norm of $\mathbf{R}^{3}$. For each point on the curve $\mathcal{C}$, there is the Frenet frame $(\mathbf{t}, \mathbf{n}, \mathbf{b})$ with respectively the tangent, normal and binormal vectors. The strength $\Gamma$ of the filament is the flux of vorticity, that is the same in each section of the filament. For a vortex ring, $S$ is the length of $\mathcal{C}$. The vortex filament may have an axial flux of strength $m$.

The thickness $\delta$ of the filament is of order $l$ and the other length scales, for example : the radius of curvature, the ring length,... are of the same order $L$. Here $l / L \ll 1$ as the vortex is slender. The parameter $\epsilon$ is defined by $\epsilon=l / L$. Here, in fact, the exact value of $l$ and $L$ are not needed.

The Reynolds number $R_{e}$ is defined as $R_{e}=\Gamma_{0} / \nu$ where $\nu$ is the kinematic viscosity of the fluid and $\Gamma_{0}$ the initial circulation of the filament. The number $\alpha$ is defined such that $R_{e}^{-1 / 2}=\alpha \epsilon$. Thus, the inviscid case is obtained when $\alpha=0$. Both inviscid $\alpha=0$ and viscous $\alpha=O(1)$ vortex filaments are studied. The Swirl number $S_{w}$ is defined as $S_{w}^{-1}=m_{0}^{*}=m_{0} /(\Gamma l)$, where $m_{0}$ is the initial axial flux of the filament. Dimensionless variables :

$$
\begin{array}{llll}
\mathbf{X}^{*} & =\mathbf{X} / L & & S^{*}=S / L \\
K^{*} & =L K & & T^{*}=L T \\
\delta^{*} & =\delta / L & t^{*} & =t /\left(L^{2} / \Gamma\right) \\
\mathbf{v}^{*} & =\mathbf{v} /(\Gamma / L) & & \boldsymbol{\omega}^{*}=\boldsymbol{\omega} /\left(\Gamma / L^{2}\right)
\end{array}
$$

are introduced. From here on, all quantities are dimensionless and the asterisks are omitted. All dimensionless variable $\mathbf{X}, \boldsymbol{\omega}, \mathbf{v}, \ldots$ have expansion in $\epsilon$. The velocity is of order $\epsilon^{-1}$, which is called leading order, then there is first order and so on.

\section{Equations of motion}

Two methods can be used to avoid the logarithmic singularity that appears in the velocity of a vortex filament without thickness : either a cut-off method [2], but it introduces an unknown length of cut off, or 
a matched asymptotic expansion of a slender vortex [1]. These both methods are described in the following and then compared.

\subsection{Cut-off methods}

The equation of motion of the central line $\mathcal{C}$ of a slender non-circular vortex ring was first expressed by different ad hoc methods. First there is Burger's method[2]

$$
\dot{\mathbf{X}}(s, t)=\frac{1}{4 \pi} \int_{I} \sigma^{\prime} \frac{\mathbf{t}\left(s^{\prime}, t\right) \times\left(\mathbf{X}(s, t)-\mathbf{X}\left(s^{\prime}, t\right)\right)}{\left|\mathbf{X}(s, t)-\mathbf{X}\left(s^{\prime}, t\right)\right|^{3}} d s^{\prime}
$$

where $\dot{\mathbf{X}}=\partial \mathbf{X} / \partial t, \sigma^{\prime}=\sigma\left(s^{\prime}, t\right)$ and $I=\left[0,2 \pi\left[\backslash\left[s-s_{c}, s+s_{c}\left[\right.\right.\right.\right.$. The unknown small variable $s_{c}$ is a small parameter introduced to avoid the singularity. This ad hoc method was called the 'cut-off method' by Crow[2], name that can be generalised to all methods that introduce a small ad hoc parameter $s_{c}$ to avoid the singularity in the integral. In this way, the slenderness of the vortex is taken into account. The parameter $s_{c}$ is called the cut-off length. In a similar way,

$$
\dot{\mathbf{X}}(s, t)=\frac{1}{4 \pi} \int_{0}^{2 \pi} \sigma^{\prime} \frac{\mathbf{t}\left(s^{\prime}, t\right) \times\left(\mathbf{X}(s, t)-\mathbf{X}\left(s^{\prime}, t\right)\right)}{\left[\left(\mathbf{X}(s, t)-\mathbf{X}\left(s^{\prime}, t\right)\right)^{2}+s_{c}^{2}\right]^{3 / 2}} d s^{\prime}
$$

can be used $[8,17,16,15,18]$. Finally, one often write[7] :

$$
\begin{gathered}
\dot{\mathbf{X}}(s, t)=\frac{1}{4 \pi} \int_{0}^{2 \pi} \sigma^{\prime} \frac{\mathbf{t}\left(s^{\prime}, t\right) \times \mathbf{M}^{\prime} \mathbf{M}}{\left|\mathbf{M}^{\prime} \mathbf{M}\right|^{3}} f\left(\frac{\left|\mathbf{M}^{\prime} \mathbf{M}\right|}{s_{c}}\right) d s^{\prime} \\
\mathbf{M}^{\prime} \mathbf{M}=\mathbf{X}(s, t)-\mathbf{X}\left(s^{\prime}, t\right)
\end{gathered}
$$

with $f(\chi) \rightarrow 1$ when $\chi \rightarrow \infty$. For example :

$$
f\left(\chi^{2}\right)=\frac{-2 \chi^{2}+\sqrt{\pi} e r f\left(\chi^{2}\right) e^{\chi^{4}}}{\sqrt{\pi} e^{\chi^{4}}}
$$

in Leonard vortex element method (VEM) [7], where erf is the error function.

The Burger's method of a non closed vortex filament is :

$$
\partial \mathbf{X} / \partial t=\frac{1}{4 \pi} \int_{I}\left[\frac{\mathbf{t}\left(s^{\prime}, t\right) \times\left(\mathbf{X}(s, t)-\mathbf{X}\left(s^{\prime}, t\right)\right)}{\left|\mathbf{X}(s, t)-\mathbf{X}\left(s^{\prime}, t\right)\right|^{3}}\right] d s^{\prime}
$$

with $I=\left[-\infty,+\infty\left[\backslash\left[s-s_{c}, s+s_{c}[\right.\right.\right.$

These methods can all be written in the common form

$$
\dot{\mathbf{X}}(s, t)=\tilde{\mathbf{Q}}\left(\mathbf{X}(s, t), s_{c}\right)
$$

where $s_{c}$ is an ad-hoc regularization parameter that has not been specified. Let us point out that here these ad-hoc regularization procedures have not been justified. 


\subsection{Asymptotic equations of motion}

The equation of motion of a non-circular slender vortex ring has been also derived from Euler or NavierStokes equations using a matched asymptotic expansion with the core thickness as a small parameter in different papers $[1,15,19,4]$. The very efficient and accurate paper on this subject is the one of Callegari and Ting[1]. These authors [1] obtained the following equation for the motion of the central line :

$$
\dot{\mathbf{X}}(s, t)=\mathbf{Q}+\frac{K(s, t)}{4 \pi}\left[-\ln \epsilon+\ln (S)-1+C_{v}(t)+C_{w}(t)\right] \mathbf{b}(s, t),
$$

with :

$$
\begin{gathered}
\mathbf{Q}=\mathbf{A}(s, t)-[\mathbf{A}(s, t) \cdot \mathbf{t}(s, t)] \mathbf{t}(s, t) \\
\mathbf{A}(s, t)=\frac{1}{4 \pi} \int_{-\pi}^{+\pi} \sigma^{\prime}\left[\frac{\mathbf{t}\left(s+s^{\prime}, t\right) \times\left(\mathbf{X}(s, t)-\mathbf{X}\left(s+s^{\prime}, t\right)\right)}{\left|\mathbf{X}(s, t)-\mathbf{X}\left(s+s^{\prime}, t\right)\right|^{3}}-\frac{K(s, t) \mathbf{b}(s, t)}{2\left|\lambda\left(s, s^{\prime}, t\right)\right|}\right] d s^{\prime} \\
\sigma^{\prime}=\sigma\left(s+s^{\prime}, t\right) \\
\lambda\left(s, s^{\prime}, t\right)=\int_{s}^{s+s^{\prime}} \sigma\left(s^{*}, t\right) d s^{*} .
\end{gathered}
$$

In this expression (9), $C_{v}(t)$ and $C_{w}(t)$ are known functions, provided the initial vorticity field is specified. This expression (9) hold for a vortex ring with axisymmetric structure at leading order and no axial variation at this order. Quick waves that evolve on a time $t / \epsilon^{2}$ and short wavelengths are also neglected. For such a vortex ring, the typical short length scale $l$ is chosen to be the initial thickness $\delta_{0}$. Here, only the leading order of the equation of motion is given. A first order is given in [15, 4, 14].

A special and interesting case is the similar vortex ring[1] for which :

$$
\begin{aligned}
& C_{v}(t)=[1+\gamma-\ln 2] / 2-\ln (\bar{\delta}) \\
& C_{w}(t)=-2\left(S_{0} / S\right)^{4}\left(m_{0} / \bar{\delta}\right)^{2} \\
& \bar{\delta}(t)=\left(S_{0} / S\right)^{1 / 2}\left(1+4 \alpha^{2} \int_{0}^{t} S\left(t^{\prime}\right) / S_{0} d t^{\prime}\right)^{1 / 2}
\end{aligned}
$$

where $\bar{\delta}=\delta / \epsilon$ and $\gamma$ is the Euler number.
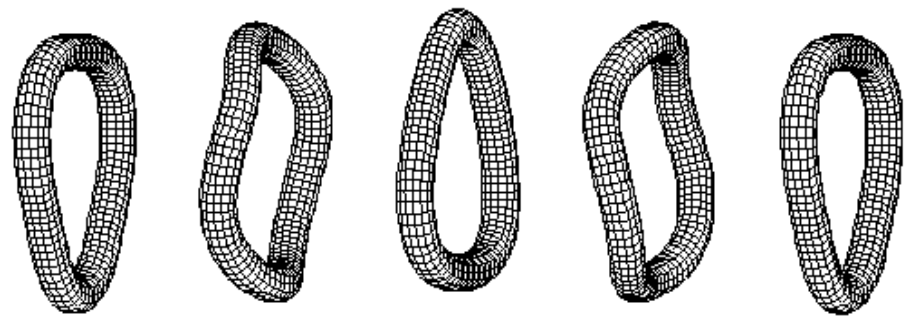

Figure 2: A perspective view of the numerical simulation of mode $n=3$ (from left to right)

A Fortran code was written to simulate numerically the equation (9) of motion of a vortex ring. An implicit finite difference scheme is used and the Simpson's rule is employed to integrate $\mathbf{Q}$. The equation of motion 
is written in a dimensionless form. The different stages of the evolution of a perturbed circular vortex ring of initial shape in a plane are found. The typical length scale $L$ is chosen to be the initial radius $R_{0}$. The second inviscid mode was investigated by Liu, Tavantziz and Ting[9]. Here, figure 2 shows the motion of a third inviscid mode with parameters $\epsilon=0.02$ and $m_{0}=0$. A linear stability study gives [13] an analytical formula for the period of oscillations as a function of the mode $n$ of the perturbation. There is good agreement between this formula and the value of periods found with numerical simulations.

For a non closed vortex filament[13], the asymptotic equation of motion is :

$$
\partial \mathbf{X} / \partial t=\mathbf{Q}+\frac{K}{4 \pi}\left(\left[\ln \frac{2}{\epsilon}-1\right]+C_{v}(t)+C_{w}(t)\right) \mathbf{b}
$$

with $\mathbf{A}(s, t)$ that is now given by :

$$
\mathbf{A}(s, t)=\frac{1}{4 \pi} \int_{-\infty}^{+\infty}\left[\frac{\mathbf{t}\left(s+s^{\prime}, t\right) \times\left(\mathbf{X}(s, t)-\mathbf{X}\left(s+s^{\prime}, t\right)\right)}{\left|\mathbf{X}(s, t)-\mathbf{X}\left(s+s^{\prime}, t\right)\right|^{3}}-H\left(1-\left|s^{\prime}\right|\right) \frac{K(s, t) \mathbf{b}(s, t)}{2\left|s^{\prime}\right|}\right] d s^{\prime}
$$

where $H$ is the Heaviside function.

\subsection{Comparison and justification of cut-off methods}

All cut-off methods introduce a cut-off length $s_{c}$, that is an unknown parameter that must be specified. The easy choice that is often done is to take it equal to the thickness $\epsilon$

$$
s_{c}=\epsilon
$$

It is interesting to notice that cut-off integrals (2-4) are singular for the small parameter that is the cut-off length $s_{c}$. Thus, these integrals can be expanded in $s_{c}$ with the matched asymptotic expansion of singular integrals method $[3,13,12]$ and the first order of this expansion can be compared with the first order asymptotic expansion in $\epsilon$ of the equation of motion (9). This gives the link between the cut-off lengths and the thickness of a viscous or inviscid vortex with an axial velocity component. It appears[11] that, at principal order, the cut-off methods are equivalent to this asymptotic equation of motion of the central curve provided that the following link between $s_{c}$ and $\epsilon$ is chosen :

$$
s_{c}(s, t)=\epsilon \frac{1}{\sigma(s, t)} e^{N-C_{v}(t)-C_{w}(t)} .
$$

The values of $N$ depend on the cut-off method that is used and are given in table 1.

\begin{tabular}{lc} 
Name of the cut-off method & $N$ \\
\hline Burger $(2)(7)$ & $1-\ln 2$ \\
Rosenhead (3) & 0 \\
VEM (4) & $1-0.009122-\ln 2$
\end{tabular}

Table 1: The parameter $N$ as a function of cut-off methods

With the use of the exact link (20) between $s_{c}$ and $\epsilon$, cut-off methods are then generalised to a viscous vortex if $\alpha \neq 0$ and the axial velocity is taken into account through $C_{w}$. As cut-off methods are equivalent to the asymptotic derivation from Navier-Stokes equations, they have the same field of validity and so $d o$ not take into account short waves. 


\section{The different regimes of a perturbed straight filament}

In dimensionless form, a perturbed straight filament is

$$
\mathbf{X}(x, t, d)=x \mathbf{t}_{0}+d \mathbf{X}^{(2)}(x, t)+o(d),
$$

where $d$ is the small amplitude of the perturbation and $x \mathbf{t}_{0}$ is the straight filament. If $L$ is the characteristic

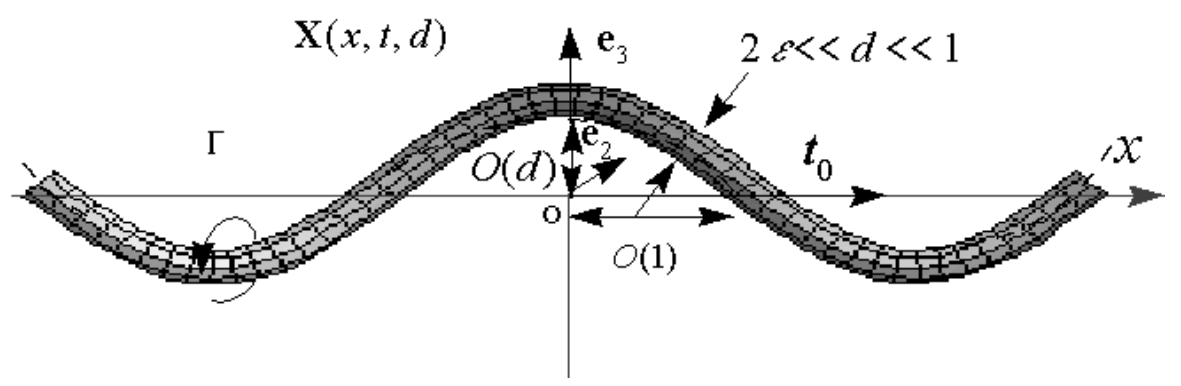

Figure 3: The straight vortex filament and its perturbation

length used to put the problem dimensionless and $\lambda$ the wavelength of the perturbation, we have chosen here $L=O(\lambda)$ to describe the filament. On the contrary, the choice $L=O(\lambda / d)$ of Klein and Majda [6] to describe the same filament gives

$$
\mathbf{X}(a, \bar{t}, d)=a \mathbf{t}_{0}+d^{2} \mathbf{X}^{(2)}\left(x=a / d, t=\bar{t} / d^{2}\right)+o\left(d^{2}\right) .
$$

Here, the perturbation wavelength is denoted $d$ and no $\epsilon$ as in Klein and Majda's paper [6] because $\epsilon$ already refers to thickness in the present work.

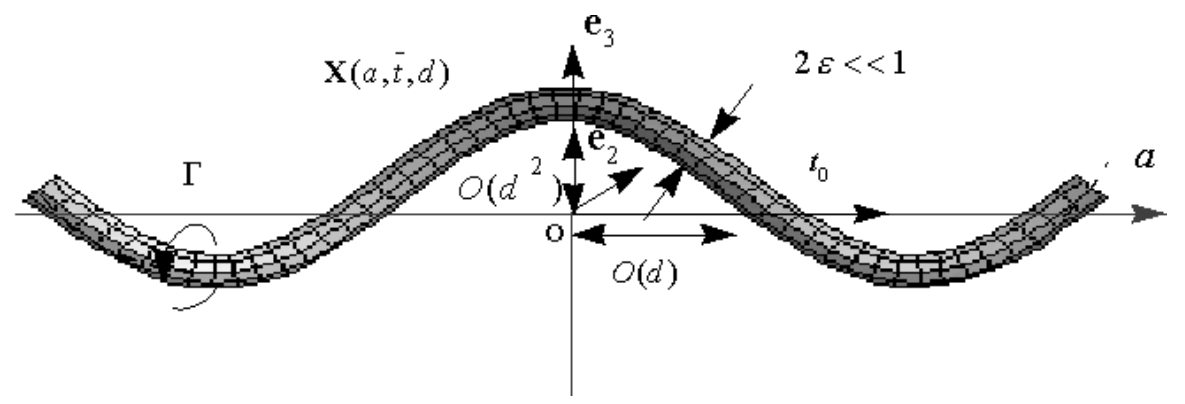

Figure 4: The filament of Klein and Majda

\subsection{Simplified equation of motion}

With the expression (21) (or its equivalent form (22)) of the central line, the equation of motion (17) simplifies $[13,10]$ in

$$
\frac{\partial \mathbf{X}}{\partial t}(x, t)=\frac{\partial \mathbf{X}^{(2)}}{\partial \bar{t}}(x, \bar{t})=\frac{1}{\tilde{\alpha}}(K \mathbf{b})(d x, t)-\left(\mathbf{t}_{0} \times \mathbf{J}(x, t)+O(d)\right)
$$

with

$$
\mathbf{J}(x, t)=\frac{1}{4 \pi} \int_{-\infty}^{+\infty}\left[\frac{\left(\mathbf{X}^{(2)}(x+h, t)-\mathbf{X}^{(2)}(x, t)\right)-h \frac{\partial \mathbf{X}^{(2)}}{\partial x}(x+h, t)}{|h|^{3}}+H(1-|h|) \frac{\frac{\partial \mathbf{X}^{(2)}}{\partial x \partial x}(x, t)}{2|h|}\right] d h
$$




$$
\begin{gathered}
\tilde{\alpha}=4 \pi /\left(\left[\ln \frac{2 d}{\epsilon}-1\right]+C_{v}+C_{w}\right) \\
(K \mathbf{b})(d x, t)=\mathbf{t}_{0} \times \mathbf{X}_{x x}^{(2)}(x, t)+O(d)
\end{gathered}
$$

and $\bar{t}=t / d^{2}$.

Hasimoto[5] introduced the following complex function $\psi$

$$
\psi=K \exp \left(i \int_{0}^{x} T d s\right)
$$

called Hasimoto's transform, where $K, T$ and $x$ are respectively the local curvature, torsion and abscise on the filament $\mathbf{X}(x, t)$. If the transformation (27) is applied to (23), this equation becomes $[6,10]$

$$
\frac{1}{i} \psi_{\tau}=\psi_{x x}+d^{2}\left(\frac{1}{2}|\psi|^{2} \psi\right)-\tilde{\alpha}(J(\psi)+O(d))
$$

where $\tau=\bar{t} / \tilde{\alpha}, \bar{t}=t / d^{2}$ and

$$
J(\psi)=\frac{1}{4 \pi} \int_{-\infty}^{+\infty}\left[\frac{(\psi(x+h, \bar{t})-\psi(x, \bar{t}))-h \frac{\partial \psi}{\partial x}(x+h, \bar{t})}{|h|^{3}}+H(1-|h|) \frac{\frac{\partial \psi}{\partial x \partial x}(x, \bar{t})}{2|h|}\right] d h .
$$

\subsection{Various degeneration of the equation of the filament function $\psi$}

The equation of motion (28) simplifies in different ways that depend on the order between the small thickness $\epsilon$ of the core (that appears in the expression of $\tilde{\alpha}$ ) and the small amplitude $d$ of the perturbation. The first interesting degeneration of the equation (28) is obtained when $\tilde{\alpha} \gg d^{2}$. The equation (28) becomes linear :

$$
\frac{1}{i} \psi_{\tau}=\psi_{x x}-\tilde{\alpha} J(\psi) \text { if } \tilde{\alpha} \gg d^{2} .
$$

The non linear term $d^{2} \frac{1}{2}|\psi|^{2} \psi$ has disappeared as it is of the same order than terms that were neglected so that the nonlocal term $\mathbf{A}(s, t, d)$ becomes $-J(\psi)$ at order 1 . The second interesting degeneration of the equation (28) is obtained when $\tilde{\alpha}=O\left(d^{2}\right)$. The equation (28) becomes :

$$
\frac{1}{i} \psi_{\tau}=\psi_{x x}+d^{2}\left(\frac{1}{2}|\psi|^{2} \psi-\kappa J(\psi)\right) \text { if } \tilde{\alpha}=O\left(d^{2}\right)
$$

with $\kappa=\tilde{\alpha} / d^{2}=O(1)$. When $\kappa=1$, this equation was first obtained by Klein and Majda[6]. Here, the local term that is in the equation of Callegari and Ting introduces a nonlinearity at the same order than the nonlocal term that becomes linear.

The third interesting degeneration of the equation (28) is obtained when $\tilde{\alpha} \ll d^{2}$. The equation (28) then becomes :

$$
\frac{1}{i} \psi_{\tau}=\psi_{x x}+d^{2}\left(\frac{1}{2}|\psi|^{2} \psi\right) \text { if } \tilde{\alpha} \ll d^{2}
$$

This is the local induction approximation for which the nonlocal integral term is negligible as regard as the local nonlinearity.

On figure 5, the various simplifications of the equation of Callegari and Ting are given according to the amplitude $d^{2}$ of the filament (21) of Klein and Majda and its thickness $\epsilon$. On this figure, $d_{s}$ is the value of the amplitude of the disturbance from which one cannot neglect any more the first nonlinear correction in the asymptotic expansion with $d$ of the nonlocal term.

It appears three zones of simplification : 
Local Induction Regime

$\frac{1}{i} \psi_{\tau}=\psi_{x x}+d^{2} \frac{1}{2}|\psi|^{2} \psi$

$\tilde{\alpha} \ll d^{2}$
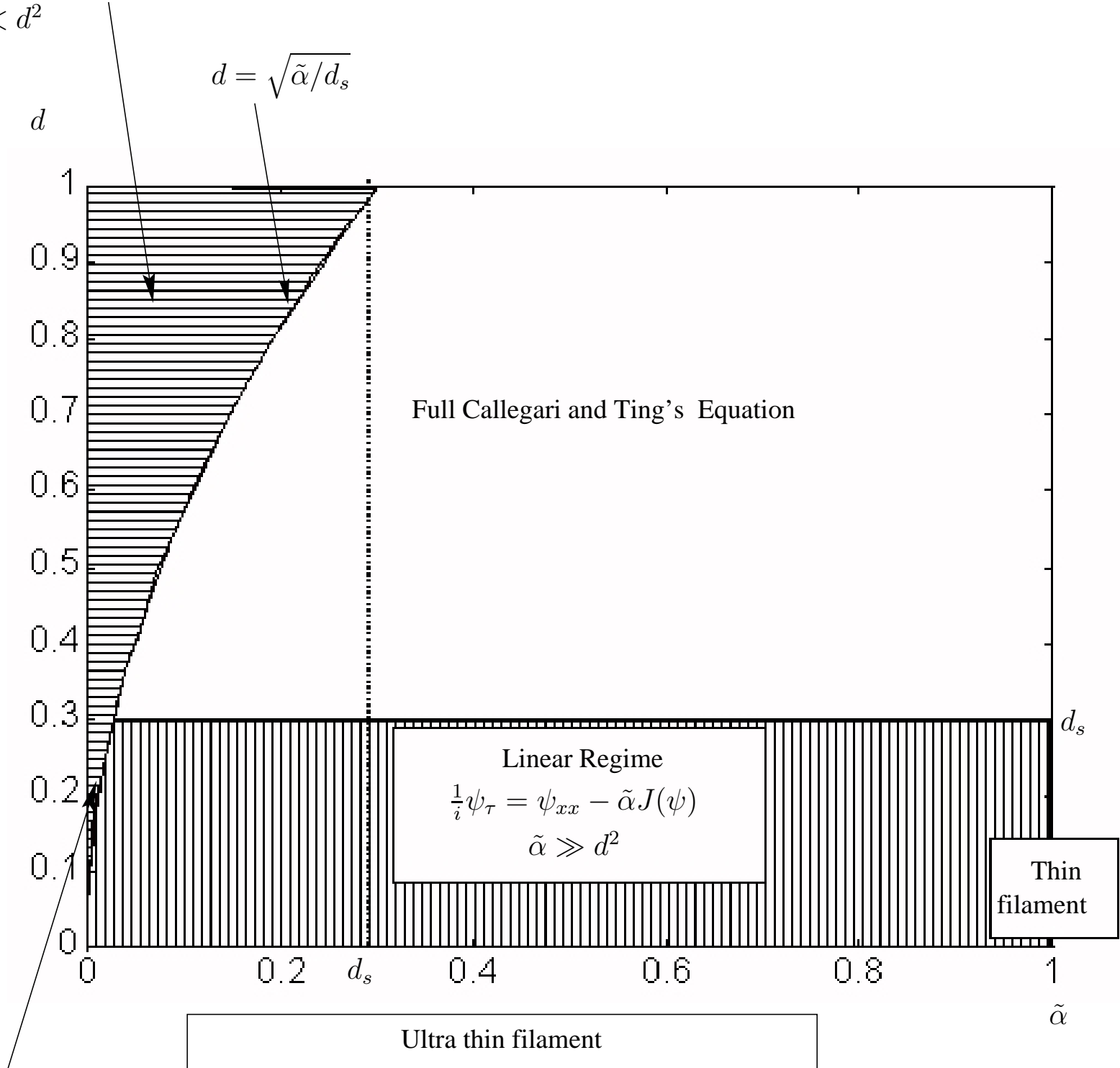

Klein and Majda Regime

$\frac{1}{i} \psi_{\tau}=\psi_{x x}+d^{2}\left(\frac{1}{2}|\psi|^{2} \psi-\kappa J(\psi)\right)$

$\tilde{\alpha}=O\left(d^{2}\right) \quad$ and $\quad \kappa=\tilde{\alpha} / d^{2}=O(1)$

Figure 5: The different simplified equations of a perturbed straight filament 
- the zone of local induction : $d>\sqrt{\tilde{\alpha} / d_{s}}$

- the zone of the regime of Klein and Majda $: d=\sqrt{\tilde{\alpha} / d_{s}}$ and $d<d_{s}$

- the linear zone $: d<\sqrt{\tilde{\alpha} / d_{s}}$ and $d<d_{s}$

The complementary zone to these three ones is the zone of the complete equation of Callegari and Ting for which one can not neglect the nonlinearity of the nonlocal term $\mathbf{A}(s, t, d)$. In these three zones, one can replace the equation of Callegari and Ting by the equation (28) and according to the zone, the equation is still simplified in one of the equations $(30,31,32)$.

When the amplitude $d^{2}$ increases, with $\tilde{\alpha}$ fixed and $\tilde{\alpha} \ll 1$, one goes gradually from the linear regime to the regime of Klein and Majda, and then to the regime of the local induction. The linearization of the regime of Klein and Majda is not the linear regime. The regime of Klein and Majda is valid only for $d=O(\sqrt{\tilde{\alpha}})$ and not for $d \ll \sqrt{\tilde{\alpha}}$ or $d \gg \sqrt{\tilde{\alpha}}$. When the amplitude $d^{2}$ increases, with $\tilde{\alpha}$ fixed and $\tilde{\alpha}=O(1)$, one goes gradually from the linear regime to the complete nonlinear regime. The regime of Klein and Majda is then never applicable when $\tilde{\alpha}=O(1)$.

\section{Conclusion}

A number of ad-hoc regularisation of the singular line Biot-Savart integral are scrutinised and compared with the results of a systematic matched asymptotic expansion of the Navier-Stokes equations. It was shown that each of these ad-hoc methods can be made to agree with the more fundamental theory by an appropriate choice of the relevant regularisation parameter, called the cut-off length. It was performed both for a vortex ring and an open vortex filament.

The equation of motion of a perturbed straight filament has been derived from the asymptotic equation of motion of an open filament. The different simplified regimes of this equation are then discussed according to both the amplitude of the perturbation and the thickness of the filament. The domains of validity of the Local Induction Approximation, the Klein and Majda regime and the lineralised Callegari and Ting equation have been then displayed on a same diagram.

\section{References}

[1] A. Callegari and L. Ting, Motion of a curved vortex filament with decaying vortical core and axial velocity, SIAM J. Appl. Math., 35 (1978), pp. 148-175.

[2] S. CRow, Stability theory for a pair of trailing vortices, AIAA J., 8 (1970), pp. 2172-2179.

[3] C. François, Les méthodes de perturbation en mécanique, ENSTA, Paris, 1981. 98-104.

[4] Y. Fukumoto and T. Mryazaki, Three dimensional distortions of a vortex filament with axial velocity, J. Fluid Mech., 222 (1991), pp. 369-416.

[5] H. Наsimoto, Motion of a vortex filament and its relation to elastica, J.Phys. Soc. Japan, 31 (1971), pp. $293-294$.

[6] R. KLein. AND A. MAJDA, Self-stretching of a perturbed vortex filament I: the asymptotic equation for derivations from a straight line, Physica D, 49 (1991), pp. 323-352.

[7] A. LeOnard, Computing three-dimensional incompressible flows with vortex elements, Ann. Rev. Fluid Mech., 17 (1985), pp. 523-559.

[8] H. Levy And A. Forsdyke, The steady motion and stability of a helical vortex, Proc. R. Soc. London A, 120 (1928), pp. 670-690.

ESAIM: Proc., Vol. 7, 1999, 278 -279 
[9] C. Liu, J. Tavantzis, and L. Ting, Numerical studies of motion and decay of vortex filaments, AIAA Journal, 24 (1986), pp. 1290-1297.

[10] D. Margerit, A note on oscillations of a straight perturbed vortex filament and on the stability of a pair of straight perturbed vortex filaments. in preparation.

[11] _ On cut-off lengths in the Biot and Savart law and the thickness of a slender vortex filament and ring, Phys. Fluids. in preparation.

[12] — The outer and inner expansions of the velocity field induced by the vorticity field of a slender vortex ring, Applied Math. Letters. in preparation.

[13] —-, Mouvement et dynamique des filaments et des anneaux tourbillons de faible épaisseur, $\mathrm{PhD}$ thesis, Institut National Polytechnique de Lorraine, november 1997.

[14] — The complete first order expansion of a slender vortex ring, in IUTAM Symposium on Dynamics of Slender Vortices, E. Krause and K. Gersten, eds., Aachen, 31 Aug.-3 Sep. 1997, Kluwer academic publishers, pp. $45-54$.

[15] D. Moore and P. Saffman, The motion of a vortex filament with axial flow, Philos. Trans. Roy. Soc. London Ser. A, 1226 (1972), pp. 403-429.

[16] P. Parks, A new look at the dynamics of vortices with finite cores, in Proc. Symposium on Aircraft Wake Turbulence, J. Olsen, A. Goldburg, and R. Rogers, eds., Seattle, Washington, 1971, Plenum Press, pp. 355-388.

[17] L. Rosenhead, The spread of vorticity in the wake behind a cylinder, Proc. R. Soc. London Ser. A, 127 (1930), pp. 590-599.

[18] P. Saffman, Vortex dynamics, Cambridge University Press, Cambridge, 1992. 33-38, 208-215.

[19] S. Widnall, D. Bliss, And A. Zalay, Theoretical and experimental study of the stability of a vortex pair, in Proc. Symposium on Aircraft Wake Turbulence, J. Olsen, A. Goldburg, and R. Rogers, eds., Seattle, Washington, 1971, Plenum Press, pp. 305-338. 